OU-HET-625

\title{
Transverse momentum distributions of quarks in the nucleon from the Chiral Quark Soliton Model
}

\author{
M. Wakamatsu ${ }^{1}$ \\ ${ }^{1}$ Department of Physics, Faculty of Science, \\ Osaka University, \\ Toyonaka, Osaka 560-0043, JAPAN
}

\begin{abstract}
We report the first calculation of the simplest but most fundamental transverse momentum dependent (TMD) distribution of quarks in the nucleon, i.e. the time-reversal-even unpolarized TMD quark and antiquark distribution with isoscalar combination, within the framework of the chiral quark soliton model. The nonperturbative account of the deformed Dirac-sea quarks within the theoretical scheme enables us to make a reliable predictions not only for the quark distribution but also for the antiquark distribution. We found that the predicted average transverse momentum square $\left\langle k_{\perp}^{2}\right\rangle$ of quarks and antiquarks depends strongly on their longitudinal momentum fraction $x$, which means that the frequently used assumption of factorization in $x$ and $k_{\perp}$ is significantly violated. It is also found, somewhat unexpectedly, that the average transverse momentum square of antiquarks is considerably larger than that of quarks.
\end{abstract}




\section{INTRODUCTION}

Growing attention has recently been paid to the transverse-momentum-dependent (TMD) parton distributions also called the unintegrated parton distributions [1]. The reason of particular interest stems from the fact that they play important roles in the theoretical description of single spin asymmetries in various hard processes including semi-inclusive deep inelastic scatterings, Drell-Yan processes, etc. [2]-[4]. The TMD parton distributions are interesting themselves also from more general viewpoint. They are expected to give a three-dimensional view of the parton distributions in momentum space, thereby providing us with complementary information besides what can be obtained through generalized parton distributions [5] -[8]].

It has been established that at the leading twist there are totally eight TMD parton distributions [9] -[11]. Among those, six are even under naive time-reversal transformations (for brevity, we call it T-even), while the remaining two are T-odd [12]-[14]. To meet the general requirement of factorization and gauge-invariance, these TMD parton distributions must be defined with the gauge link operators called Wilson lines [15] -[20]. Since these Wilson lines, which can be interpreted as simulating the effects of gluon initial and final state interactions, are process dependent [16], the TMD parton distributions are in general non-universal [21],[22]. Still, a remarkable difference between the T-even and T-odd TMD parton distributions should not be overlooked. For the former, the presence of the Wilson lines is less crucial in the sense that they do survive even without such gauge links. On the contrary, for the T-odd TMD parton distributions, the introduction of the Wilson lines is of fatal importance since they do vanish identically in the absence of such effects of initial and final state interactions [15],[16]. The existence of the former types of TMD distributions appears only natural since the intrinsic motion of quarks described by the naive bound state wave function without any complex phase is likely to be three dimensional and it should be described by some TMD distribution function.

Unlike the integrated parton distributions, our knowledge on the TMD parton distributions is very poor. Very recently, through the analysis of azimuthal asymmetries both in hadron-hadron collisions and in semi-inclusive deep-inelastic scatterings, Anselmino et al. extracted a T-odd TMD distribution $f_{1 T}^{\perp}$ called the Sivers function [23]. There also exists an attempt to extract another T-odd distribution called the Boer-Mulders function [24]. In 
these studies, however, a flavor independent Gaussian distribution of the transverse momentum under the factorized ansatz in the variables $x$ and $k_{\perp}$ is assumed by reason of simplicity, although there is no compelling reason for this choice. Since enough empirical information to test such assumption cannot be expected at the present moment, any information from models of baryons and/or the lattice QCD would be extremely valuable.

Although there exist a lot of model calculations for the integrated parton distributions, there are not so many for the TMD distributions. (See [25] for a compact overview of those studies.) The leading-twist T-even functions were calculated in a spectator model with scalar and axial-vector quarks [26], and in a light-cone quark model where the Fock expansion is truncated to consider only 3 valence quarks [27]. On the other hand, the T-odd functions are investigated in the spectator model with scalar diquarks [28],|29] and with scalar and axial-vector diquarks [30], [31], [25] in the MIT bag model [32] -[34], and in the constituent quark model [35]. Very recently, the first lattice QCD calculation of the lowest moments of the TMD quark distributions $f_{1}\left(x, \boldsymbol{k}_{\perp}\right)$ and $g_{1 T}\left(x, \boldsymbol{k}_{\perp}\right)$ has also been reported [36]. (Although our main interest here is concerned with the TMD quark distributions inside the nucleon, we recall that there also exist some investigations on the TMD distributions in the pseudoscalar mesons including the pion based on the SU(3) Nambu-Jona-Lasinio model [37] -[39].)

Now, the purpose of the present paper is to evaluate the simplest but most fundamental TMD parton distributions in the nucleon, i.e. the T-even unpolarized TMD quark and antiquark distributions with isoscalar combination, within the framework of the chiral quark soliton model (CQSM) [40],[41]. The greatest advantage of the CQSM over the other models of baryons is its full account of nonperturbative chiral dynamics of QCD, which has been proven to be essential for the physics of light quark distribution functions [42] -[48]. The nonperturbative account of the deformed Dirac-sea quarks within the scheme enables us to make a reliable predictions not only for the quark distributions but also for the antiquark distributions, as already been proven through its good reproduction of the famous NMC (New Muon Collaboration) observation, i.e. the dominance of the $\bar{d}$ sea over the $\bar{u}$ one in the proton, etc. [49], [46], [50]. We therefore expect that the same model would provide us with valuable and reliable information also on the nature of the transverse motion of quarks and antiquarks inside the nucleon.

The plan of the paper is as follows. First, in sect.II, we derive theoretical formulas 
necessary for the calculation of the TMD quark and antiquark distributions within the framework of the CQSM. Next, in sect.III, the predictions of the CQSM are shown and the detailed discussion on them will be given. Conclusion of our analysis is then given in sect.IV.

\section{UNPOLARIZED TMD QUARK DISTRIBUTIONS}

We are interested here in the simplest but most fundamental TMD parton distribution function, i.e. the unpolarized TMD quark distribution. The unpolarized TMD quark distribution function of flavor $a$ in the nucleon, averaged over its spin, is defined as

$$
f^{a}\left(x, \boldsymbol{k}_{\perp}\right)=\left.\int \frac{d \xi^{-} d^{2} \boldsymbol{\xi}_{\perp}}{2(2 \pi)^{3}} e^{i k \cdot \xi}\left\langle P\left|\bar{\psi}_{a}(0)\left(1+\gamma^{0} \gamma^{3}\right) \mathcal{U}_{[0, \xi]} \psi_{a}(\xi)\right| P\right\rangle\right|_{\xi^{+}=0},
$$

where

$$
\mathcal{U}_{[0, \xi]} \equiv \mathcal{P} e^{-i g \int_{0}^{\xi} A(w) \cdot d w}
$$

is the so-called gauge link operator, also called Wilson line, connecting the two different space-time points 0 and $\xi$, by all possible ordered path. This gauge link is known to simulate the interaction of the outgoing quark field with the spectators inside the hadron, and it works to ensure the color gauge invariance of the above definition of the TMD quark distribution [16].

Here, we want to evaluate the TMD quark distribution within the framework of the CQSM. Since the CQSM is an effective quark theory, which does not contain gluonic degrees of freedom at least explicitly, we can drop the gauge link operator. As a matter of course, this simple procedure is fatal for predicting the T-odd TMD distributions like the Sivers function [12], [13] or the Boer-Mulders function [14], since the complex phase arising from the gauge link is indispensable for the existence of such T-odd distribution functions. Roughly speaking, the T-even distribution function we are to calculate below can be thought of as a "static" TMD quark distribution, which can be computed directly from the bound state wave functions of a target nucleon without accompanying complex phase arising from the effects of final state interactions [51].

Though the definition (1) of the TMD distribution function itself is Lorentz-frame independent, it is convenient to evaluate it in the nucleon rest frame, where $\boldsymbol{P}=0$ and $P_{0}=M_{N}$ with $M_{N}$ being the nucleon mass. This enables us to rewrite the exponential factor $e^{i k \cdot \xi}$ 
contained in (11) in the following manner :

$$
e^{i k \cdot \xi}=e^{i k_{+} \xi^{-}-i \boldsymbol{k}_{\perp} \cdot \boldsymbol{\xi}_{\perp}}=e^{i x P_{+} \xi^{-}-i \boldsymbol{k}_{\perp} \cdot \boldsymbol{\xi}_{\perp}}=e^{i x M_{N} \xi^{0}-i \boldsymbol{k}_{\perp} \cdot \boldsymbol{\xi}_{\perp}}
$$

Here, we have used the relation $\xi^{3}=-\xi^{0}$ (or $\xi^{+}=0$ ), together with the standard definition of the light-cone vector, $\xi^{ \pm}=\left(\xi^{0} \pm \xi^{3}\right) / \sqrt{2}$.

To proceed further, we notice that the large- $N_{c}$ behavior of the unpolarized TMD quark distribution depends on the isospin combination [52]. Just similarly to the integrated unpolarized distributions extensively studied before [42]-[44], [46],[48], the isoscalar distribution is dominant in the large- $N_{c}$ limit, and survives at the mean-field level, while the isovector combination appears as the first order correction in the collective angular velocity operator $\Omega$ of the rotating soliton. (We recall that $\Omega$ is an $1 / N_{c}$ quantity.) Since the evaluation of the $O\left(\Omega^{1}\right)$ contribution is much harder, let us concentrate on the calculation of the isoscalar part in the present paper. Using the formalism developed in the previous studies [42] -[48], we find that the isoscalar unpolarized TMD quark distribution $f^{u+d}\left(x, \boldsymbol{k}_{\perp}\right)$ in the CQSM is given in the following form :

$$
\begin{aligned}
f^{u+d}\left(x, \boldsymbol{k}_{\perp}\right)=N_{c} & M_{N} \int d^{3} \boldsymbol{R} \int \frac{d \xi^{0} d^{2} \boldsymbol{\xi}_{\perp}}{(2 \pi)^{3}} e^{i x M_{N} \xi^{0}-i \boldsymbol{k}_{\perp} \cdot \xi_{\perp}} \\
& \times\left.\sum_{n \in o c c} e^{i E_{n} \xi^{0}} \Phi_{n}^{\dagger}(-\boldsymbol{R})\left(1+\alpha^{3}\right) \Phi_{n}(\boldsymbol{\xi}-\boldsymbol{R})\right|_{\xi^{3}=-\xi^{0}},
\end{aligned}
$$

with $\alpha^{3}=\gamma^{0} \gamma^{3}$. Here, $\Phi_{n}(\boldsymbol{x})$ is the eigenfunctions of the Dirac hamiltonian $H$ with the corresponding eigenvalue $E_{n}$, i.e.

$$
H \Phi_{n}(\boldsymbol{x})=E_{n} \Phi_{n}(\boldsymbol{x})
$$

with

$$
H=\frac{\boldsymbol{\alpha} \cdot \nabla}{i}+M \beta e^{i \gamma_{5} \boldsymbol{\tau} \cdot \hat{\boldsymbol{r}} F(r)} .
$$

In (41), sum $_{n \in \text { occ }}$ denotes the summation over the occupied states in the hedgehog mean field. In deriving (4), the projection into a nucleon state with given momenta $\boldsymbol{P}$ has been achieved, as usual, by integrating over all shift of the center-of-mass coordinate $\boldsymbol{R}$ of the soliton,

$$
\left\langle\boldsymbol{P}^{\prime}|\cdots| \boldsymbol{P}\right\rangle=\int d^{3} \boldsymbol{R} e^{i\left(\boldsymbol{P}^{\prime}-\boldsymbol{P}\right) \cdot \boldsymbol{R}} \cdots
$$

Introducing the eigenfunctions in the momentum representation,

$$
\Phi_{n}(\boldsymbol{x})=\int \frac{d^{3} \boldsymbol{p}}{(2 \pi)^{3}} e^{i \boldsymbol{p} \cdot \boldsymbol{x}} \tilde{\Phi}_{n}(\boldsymbol{p}),
$$


(41) can readily be transformed into the form :

$$
\begin{aligned}
f^{u+d}\left(x, \boldsymbol{k}_{\perp}\right)= & N_{c} M_{N} \int \frac{d \xi^{0} d^{2} \boldsymbol{\xi}_{\perp}}{(2 \pi)^{3}} e^{i x M_{N} \xi^{0}-i \boldsymbol{k}_{\perp} \cdot \boldsymbol{\xi}_{\perp}} \\
& \times \sum_{n \in o c c} e^{i E_{n} \xi^{0}} \int \frac{d^{3} \boldsymbol{p}}{(2 \pi)^{3}} e^{-i \boldsymbol{p} \cdot \boldsymbol{\xi}} \tilde{\Phi}_{n}^{\dagger}(\boldsymbol{p})\left(1+\alpha_{3}\right) \tilde{\Phi}_{n}(\boldsymbol{p}) .
\end{aligned}
$$

Now, by noting that $e^{i \boldsymbol{p} \cdot \boldsymbol{\xi}}=e^{i p^{3} \xi^{3}} e^{i \boldsymbol{p}_{\perp} \cdot \boldsymbol{\xi}_{\perp}}=e^{-i p^{3} \xi^{0}} e^{i \boldsymbol{p}_{\perp} \cdot \boldsymbol{\xi}_{\perp}}$, one can carry out the integration over $\xi^{0}$ and $\boldsymbol{\xi}_{\perp}$ to obtain

$$
\begin{aligned}
f^{u+d}\left(x, \boldsymbol{k}_{\perp}\right)= & M_{N} N_{c} \int \frac{d^{3} \boldsymbol{p}}{(2 \pi)^{3}} \\
& \times \sum_{n \in o c c} \tilde{\Phi}_{n}^{\dagger}(\boldsymbol{p})\left(1+\alpha^{3}\right) \delta\left(x M_{N}-E_{n}-p^{3}\right) \delta^{2}\left(\boldsymbol{p}_{\perp}-\boldsymbol{k}_{\perp}\right) \tilde{\Phi}_{n}(\boldsymbol{p}) .
\end{aligned}
$$

As usual, the numerical evaluation of the above expression is carried out by using the discretized momentum basis of Kahana and Ripka [53], [54], so that it is convenient to introduce the smeared distribution defined by [43]

$$
f_{\gamma}^{u+d}\left(x, \boldsymbol{k}_{\perp}\right) \equiv \frac{1}{\sqrt{\pi} \gamma} \int_{-\infty}^{\infty} d x^{\prime} e^{-\frac{\left(x-x^{\prime}\right)^{2}}{\gamma^{2}}} f^{u+d}\left(x, \boldsymbol{k}_{\perp}\right) .
$$

After some straightforward algebra, we therefore get

$$
f_{\gamma}^{u+d}\left(x, \boldsymbol{k}_{\perp}\right)=N_{c} \sum_{n \in o c c} \int_{-\infty}^{\infty} d k_{3} e^{-\frac{1}{\gamma^{2}}\left(x-\frac{E_{n}+k_{3}}{M_{N}}\right)^{2}} \tilde{\Phi}_{n}^{\dagger}\left(\boldsymbol{k}_{\perp}, k_{3}\right)\left(1+\alpha^{3}\right) \tilde{\Phi}_{n}\left(\boldsymbol{k}_{\perp}, k_{3}\right) .
$$

Here, we have changed the integration variable from $p^{3}$ to $k_{3}$. We recall now that $\tilde{\Phi}_{n}$ is simultaneous eigenfunctions of the hamiltonian $H$, and the grand spin operator $\boldsymbol{K}=\boldsymbol{J}+\frac{1}{2} \boldsymbol{\tau}$ and its projection $M_{K}$ on the z-axis. The functions $\tilde{\Phi}_{n}$ can be expanded in terms of the discretized plane-wave basis of Kahana and Ripka as

$$
\tilde{\Phi}_{n}=\sum_{\alpha} \sum_{i} c_{\alpha i}^{(n)} \phi_{\alpha i}
$$

where the index $\alpha$ distinguishes 4 independent plane-wave basis with definite grand spin $K, M_{K}$, and parity. (We point out that the expansion coefficients $c_{\alpha i}^{(n)}$ can be chosen real numbers.) On the other hand, $i$ labels the discretized momenta $k_{i}$, which is determined by imposing the boundary condition

$$
j_{K}\left(k_{i} D\right)=0
$$

at the radius $r=D$ chosen to be sufficiently larger than the typical soliton size. (The number of momentum bases is made finite by introducing the maximum momentum $k_{\max }$ 
such that $k_{i}<k_{\max }$.) Since the Kahana-Ripka plane-wave basis is given in the spherical representation, we also express $\boldsymbol{k}_{\perp}$ and $k_{3}$ in the spherical coordinates as

$$
\begin{aligned}
\left(\boldsymbol{k}_{\perp}\right)_{x} & =k_{i} \sin \theta \cos \phi \\
\left(\boldsymbol{k}_{\perp}\right)_{y} & =k_{i} \sin \theta \sin \phi, \\
k_{3} & =k_{i} \cos \theta .
\end{aligned}
$$

Since the final answer for the TMD distribution $f^{u+d}\left(x, \boldsymbol{k}_{\perp}\right)$ should be independent of the azimuthal angle $\phi$ of the transverse momentum $\boldsymbol{k}_{\perp}$, the following replacement is justified

$$
\phi_{\alpha i}^{\dagger}\left(1+\alpha_{3}\right) \phi_{\beta j} \longrightarrow \int \frac{d \phi}{2 \pi} \phi_{\alpha i}^{\dagger}\left(1+\alpha_{3}\right) \phi_{\beta j} .
$$

The result of $\phi$ integration can schematically be written as

$$
\int \frac{d \phi}{2 \pi} \phi_{\alpha i}^{\dagger}\left(1+\alpha_{3}\right) \phi_{\beta j}=\delta_{i j} \mathcal{F}_{\alpha \beta}\left(k_{i}, \cos \theta\right)
$$

where $\mathcal{F}_{\alpha \beta}\left(k_{i}, \cos \theta\right)$ is a function of $k_{i}$ and $\cos \theta$. The Kronecker delta $\delta_{i j}$ arises from the fact that the operator $\left(1+\alpha_{3}\right)$ does not change momentum. Because the magnitude of the transverse momentum $k_{\perp} \equiv\left|\boldsymbol{k}_{\perp}\right|$ is given externally, the relations (16)-(17) dictates that, once the plane-wave-basis momentum $k_{i}$ is given, both of $k_{3}$ and $\cos \theta$ are fixed as

$$
k_{3}= \pm \sqrt{k_{i}^{2}-k_{\perp}^{2}} \equiv k_{3, i}, \quad \cos \theta=\frac{k_{3}}{k_{i}} \equiv \cos \theta_{i}
$$

We therefore approximate the expression (11) as

$$
f_{\gamma}^{u+d}\left(x, \boldsymbol{k}_{\perp}\right)=N_{c} \sum_{n \in o c c} \sum_{\alpha, \beta} \sum_{i} c_{\alpha i}^{(n)} c_{\beta i}^{(n)} \mathcal{F}_{\alpha \beta}\left(k_{i}, \cos \theta_{i}\right) \frac{1}{\sqrt{\pi} \gamma} e^{-\frac{1}{\gamma^{2}}\left(x-\frac{E_{n}+k_{3, i}}{M_{N}}\right)^{2}} .
$$

In the next section, we show that this algorithm in fact works if we take large enough boundary radius $D$, at least for the calculation of Dirac sea contributions. Unfortunately, we find that the same algorithm does not work very well for the calculation of the discrete valence level contribution (corresponding to $n=0$ ). This causes no serious problem, however, since we can use simpler method to evaluate this part. We just go back to the expression (10) before introducing smeared distribution, and obtain

$$
f_{v a l}^{u+d}\left(x, \boldsymbol{k}_{\perp}\right)=M_{N} N_{c} \tilde{\Phi}_{0}^{\dagger}\left(\boldsymbol{k}_{\perp}, k_{3}=x M_{N}-E_{0}\right)\left(1+\alpha_{3}\right) \tilde{\Phi}_{0}\left(\boldsymbol{k}_{\perp}, k_{3}=x M_{N}-E_{0}\right)
$$

where $\tilde{\Phi}_{0}$ and $E_{0}$ are the eigenfunction and the eigenenergy corresponding the valence level. Since $\tilde{\Phi}_{0}$ is a discrete bound state wave function anyhow, we can evaluate the above expression without any difficulty by using its momentum space wave function as used in [45]. 


\section{NUMERICAL RESULTS AND DISCUSSIONS}

The basic lagrangian of the model contains two physical parameters, the weak pion decay constant $f_{\pi}$, the dynamically generated effective quark mass $M$. As usual, $f_{\pi}$ is fixed to be its physical value, i.e. $f_{\pi}=93 \mathrm{MeV}$. On the other hand, $M$ is taken to be $375 \mathrm{MeV}$, which is favored from our previous analysis of the nucleon spin structure functions [47], [48].

The model contains ultraviolet divergences so that it must be regularized by introducing some physical cutoff. Following the previous studies, we simply use the Pauli-Villars regularization scheme with single subtraction. In this scheme, any nucleon observables including quark distribution functions in the nucleon are regularized through the subtraction [43] :

$$
\langle O\rangle^{r e g} \equiv\langle O\rangle^{M}-\left(\frac{M}{M_{P V}}\right)^{2}\langle O\rangle^{M_{P V}} .
$$

Here $\langle O\rangle^{M}$ denotes the nucleon matrix element of an operator $O$ evaluated with the original effective action with the mass parameter $M$, while $\langle O\rangle^{M_{P V}}$ stands for the corresponding matrix element obtained from $\langle O\rangle^{M}$ by replacing the parameter $M$ with the Pauli-Villars cutoff mass $M_{P V}$. Demanding that the regularized action reproduces the correct normalization of pion kinetic term in the corresponding bosonized action, $M_{P V}$ is uniquely fixed by the relation

$$
\frac{N_{c}}{4 \pi^{2}} M^{2} \log \frac{M_{P V}^{2}}{M^{2}}=f_{\pi}
$$

For $M=375 \mathrm{MeV}$, this gives $M_{P V} \simeq 562 \mathrm{MeV}$, leaving no adjustable parameter.

A short comment may be necessary for the regularization scheme explained above. As was shown in [55], the Pauli-Villars scheme with a single subtraction term is not a completely satisfactory regularization procedure. It fails to remove ultraviolet divergences of some special quantities like the vacuum quark condensate, which contains quadratic divergence instead of logarithmic one. For obtaining finite answers also for these special observables, the single-subtraction Pauli-Villars scheme is not enough. It was shown that more sophisticated Pauli-Villars scheme with two subtraction terms meets this requirement [55]. Fortunately, the self-consistent solution of the CQSM obtained in this double-subtraction Pauli-Villars scheme is only slightly different from that of the naive single-subtraction scheme, except when dealing with some special quantities containing quadratic divergences [55]. Considering the fact that the calculation of quark distribution functions, much more the TMD quark distribution functions, in the CQSM, is extremely time-consuming and that the most nucleon 
observables are rather insensitive to which regularization scheme is chosen, we shall simply use here the single-subtraction Pauli-Villars scheme. (The use of more time-consuming double subtraction scheme is mandatory, however, for some special parton distribution functions containing quadratic divergences. An example is the chiral-odd twist-3 unpolarized distribution functions $e(x)$ investigated in [56] -[59].)
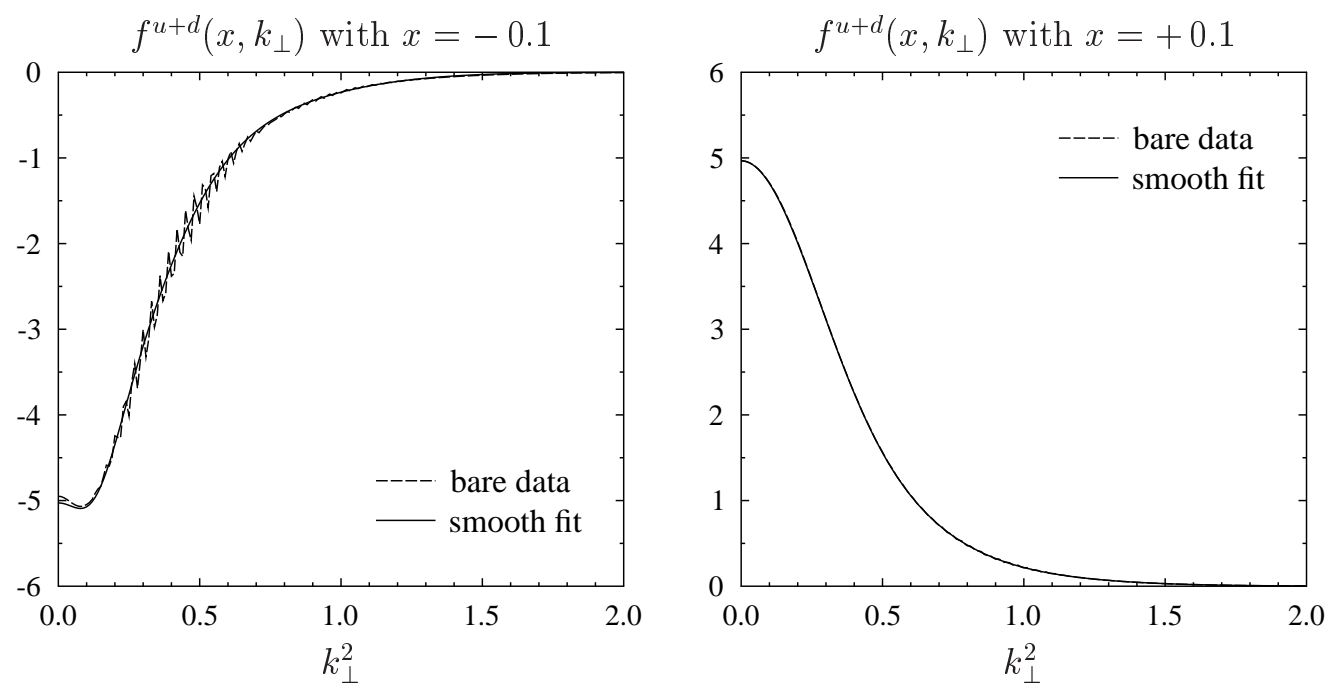

FIG. 1: The Dirac-sea contributions to the unpolarized TMD distribution with isoscalar combination $f^{u+d}\left(x, \boldsymbol{k}_{\perp}\right)$ for two typical values of $x$, i.e. $x=-0.1$ (left panel), and $x=+0.1$ (right panel). In both figures, the dashed curves represent the bare numerical predictions of the model obtained with $D=40 / M, k_{\max }=10 M$, and $\gamma=0.3$, while the solid curves are their smooth fits. Note that the dashed and solid curves are almost indistiguishable in the right panel.

First, let us check whether the numerical algorithm proposed in the previous section in fact works. As pointed out there, there is no difficulty in the calculation of discrete valence level contribution to the TMD quark distribution, so that we concentrate on the Dirac-sea contributions. Shown in Fig 1 are the predictions of the CQSM for the Dirac-sea contributions to $f^{u+d}\left(x, \boldsymbol{k}_{\perp}\right)$ for two typical values of longitudinal momentum fraction, i.e. $x=-0.1$ (left panel) and $x=+0.1$ (right panel). In these figures, the dashed curves are the bare numerical predictions of the model obtained with $D=40 / M, k_{\max }=10 M$, and $\gamma=0.03$. The observed fluctuating behavior, which is a little stronger for the negative value of $x$, is due to the use of the discretized basis with fairly small $\gamma$. We fit these results with smooth function shown by solid curves. 
After demonstrating that our numerical method in fact works as long as one takes large enough boundary radius $D$, let us now show the full CQSM predictions for the unpolarized TMD quark and antiquark distribution functions. We recall that the function $f^{u+d}\left(x, \boldsymbol{k}_{\perp}\right)$ with positive $x$ can literally be interpreted as quark distribution, whereas the function with negative $x$ should be interpreted as antiquark distributions with an extra minus sign as

$$
f^{u+d}\left(-x, \boldsymbol{k}_{\perp}\right)=-f^{\bar{u}+\bar{d}}\left(x, \boldsymbol{k}_{\perp}\right), \quad(0<x<1) .
$$

Fig 2 show the CQSM predictions for $f^{u+d}\left(x, \boldsymbol{k}_{\perp}\right)$ as functions of $k_{\perp}^{2}$ for 6 typical values of $x$, i.e. $x=1.0 \times 10^{-6}, 0.1,0.2,0.4,0.6$ and 0.8. On the other hand, Fig. 3 shows $f^{u+d}\left(x, \boldsymbol{k}_{\perp}\right)$ for 6 different values of $x$, i.e. $x=-0.8,-0.6,-0.4,-0.2,-0.1$ and $-1.0 \times 10^{-6}$. In these figures, the contributions of the discrete valence-level quarks and those of the Dirac-sea quarks are illustrated by the dashed and dash-dotted curves, respectively, while their sums are denoted by the solid curves. First, let us look into the quark distribution with $x>0$. As expected, the contributions of Dirac-sea quark is most important in the lower $x$ region, while the contribution from the discrete valence level dominates over that from the Dirac-sea quarks as $x$ increases to approach 1 . Somewhat unexpectedly, the contributions from the Dirac-sea quarks are seen to have longer range tail in $k_{\perp}$ space, as most clearly seen from the figure corresponding to $x=0.1$. (The plot of $f^{u+d}\left(x, \boldsymbol{k}_{\perp}\right)$ is not the best way to see this unique feature. A better way to see it more clearly would be to examine the graph of the $k_{\perp}$-weighted distribution $k_{\perp} f^{u+d}\left(x, \boldsymbol{k}_{\perp}\right)$, the integral of which over $k_{\perp}$ gives the integrated quark distribution $f^{u+d}(x)$. See the discussion later, for more detail.) 
$f^{u+d}\left(x, k_{\perp}^{2}\right)$ in the region $x>0$
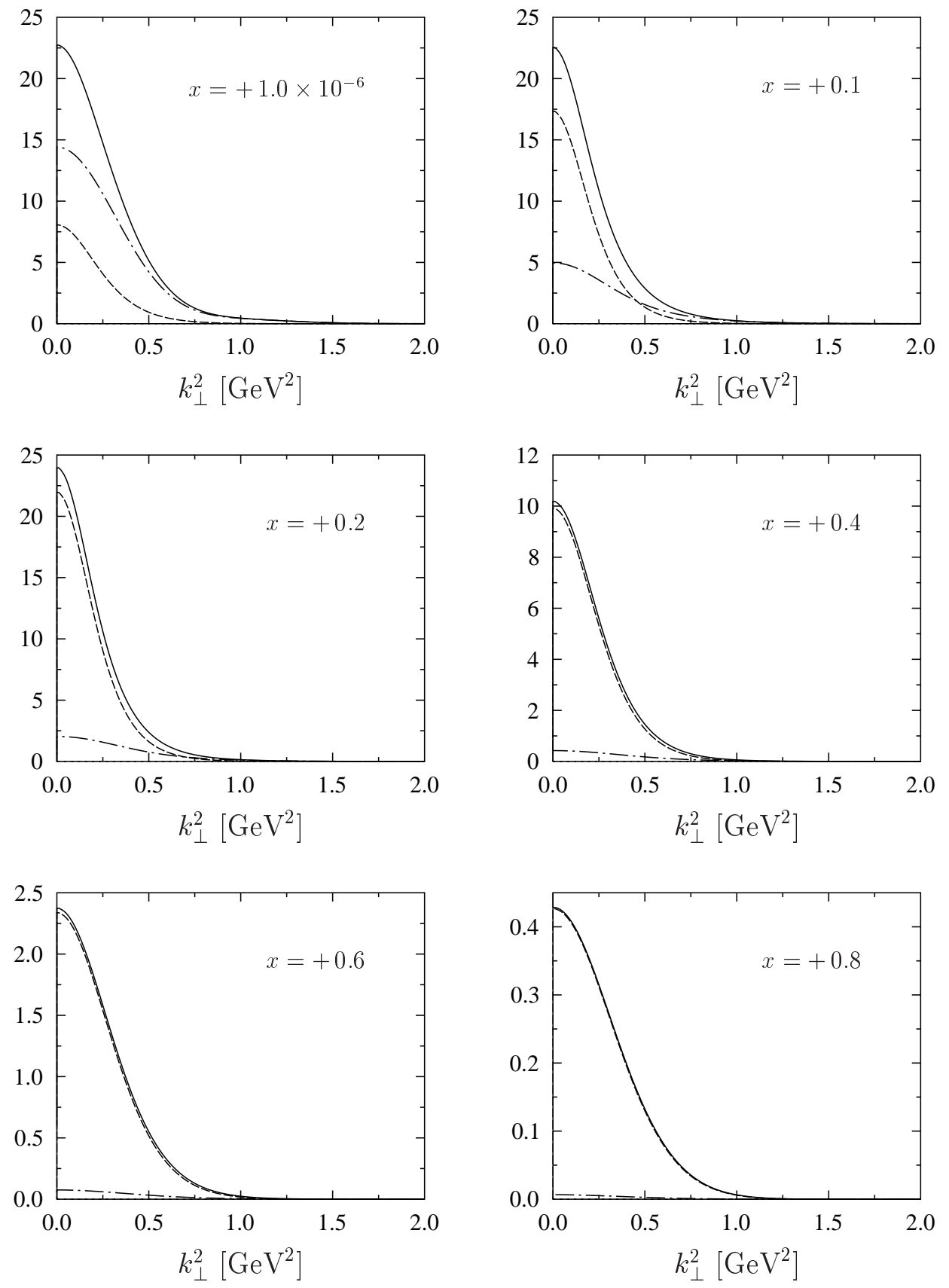

FIG. 2: The CQSM predictions for the unpolarized TMD distribution with isoscalar combination $f^{u+d}\left(x, \boldsymbol{k}_{\perp}\right)$ in the positive $x$ region. They are shown for 6 typical values of $x$, i.e. $x=1.0 \times$ $10^{-6}, 0.1,0.2,0.4,0.6$ and 0.8 . In these figures, the dashed and dash-dotted curves respectively stand for the contributions of the discrete valence level and of the deformed Dirac-sea quarks, while their sum is represented by the solid curves. 
$f^{u+d}\left(x, k_{\perp}^{2}\right)$ in the region $x<0$
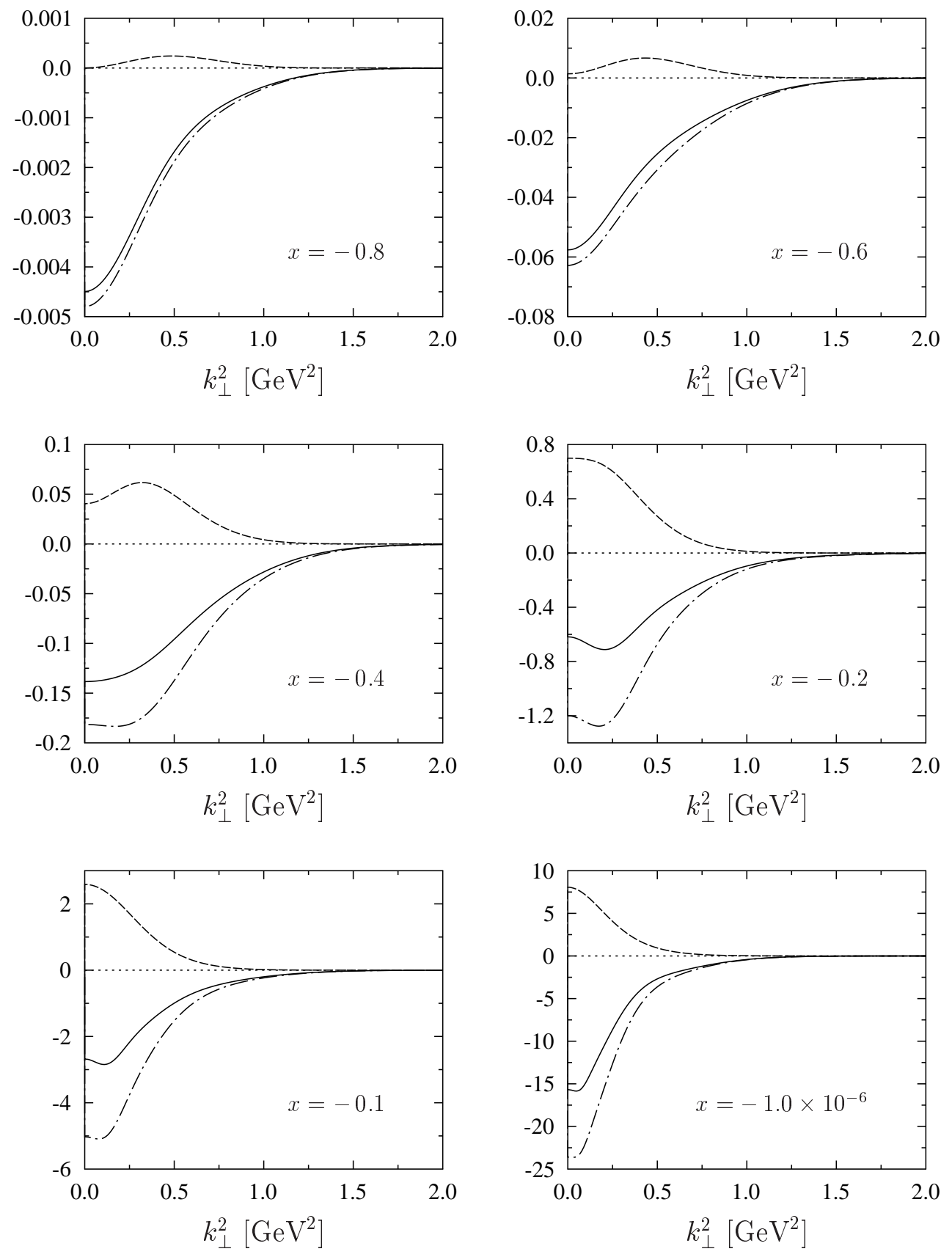

FIG. 3: The CQSM predictions for the unpolarized TMD distribution with isoscalar combination $f^{u+d}\left(x, \boldsymbol{k}_{\perp}\right)$ in the negative $x$ region, which correspond to the antiquark distributions, according to the rule $f^{\bar{u}+\bar{d}}\left(x, \boldsymbol{k}_{\perp}\right)=-f^{u+d}\left(-x, \boldsymbol{k}_{\perp}\right)$ with $0<x<1$. They are shown for 6 typical values of $x$, i.e. $-0.8,-0.6,-0.4,-0.2,-0.1$ and $-1.0 \times 10^{-6}$. The meaning of the curves is the same as in Fig,2, 
Turning next to the distribution with $x<0$, corresponding to the antiquark distribution, we find that the contributions from the Dirac-sea quarks dominate over that of valence level. Here, the fact that the Dirac-sea contributions have higher $k_{\perp}$ components than the valence-level quark is much more evidently seen. An importance notice here is that the total contributions shown by the solid curves are all negative for any $x$. In consideration of the extra minus sign indicated in (25), this means that the positivity of the TMD antiquark distribution $f^{\bar{u}+\bar{d}}\left(x, \boldsymbol{k}_{\perp}\right)$ is legitimately fulfilled in the CQSM. We emphasize that the proper inclusion of the Dirac-sea contribution is crucial for the fulfillment of this fundamental property of unpolarized parton distribution. It is this unique feature of the CQSM that enables us to make a reasonable predictions not only for the quark distribution but also the antiquark (or the sea quark) distributions.
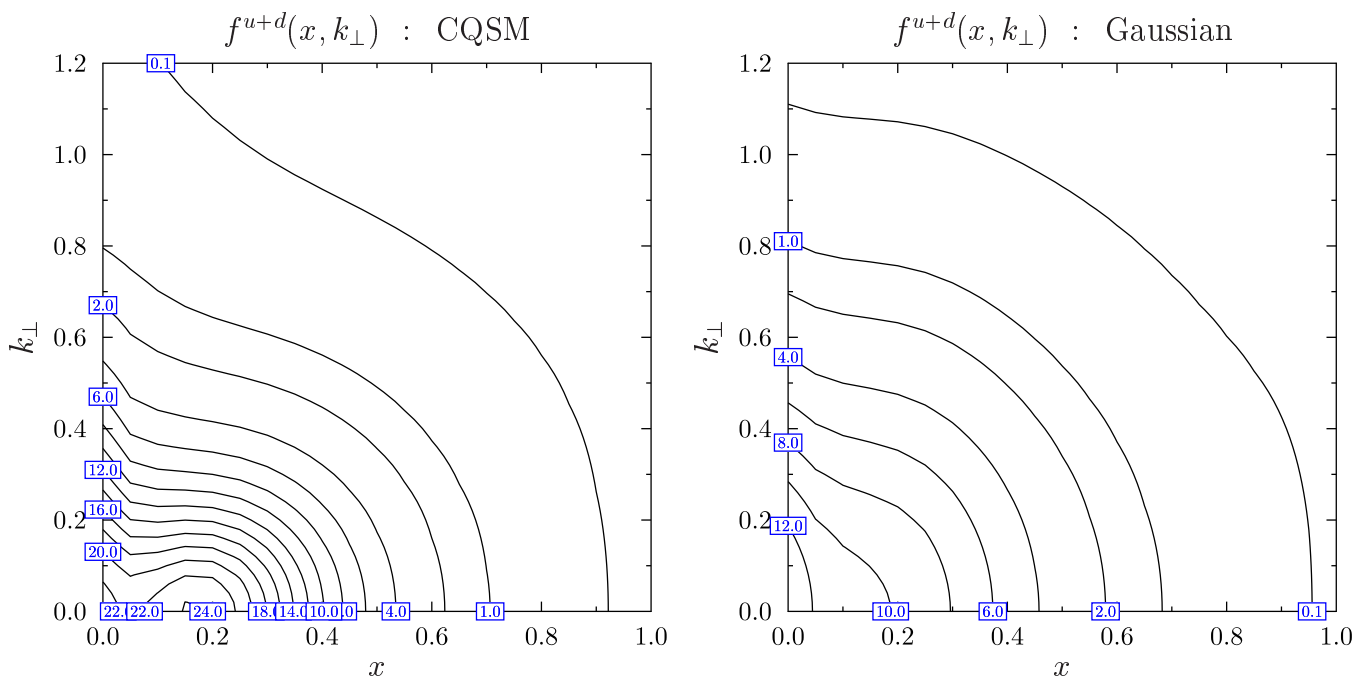

FIG. 4: The contour plot for the isoscalar unpolarized TMD quark distribution function $f^{u+d}\left(x, \boldsymbol{k}_{\perp}\right)$. The left panel corresponds to the prediction of the CQSM, while the right panel to the schematic distribution in the factorized form $f^{u+d}\left(x, \boldsymbol{k}_{\perp}\right)=f^{u+d}(x) \frac{1}{\pi\left\langle k_{\perp}^{2}\right\rangle} e^{-k_{\perp}^{2} /\left\langle k_{\perp}^{2}\right\rangle}$ with $\left\langle k_{\perp}^{2}\right\rangle=0.25 \mathrm{GeV}^{2}$.

For lack of enough empirical information, the TMD quark distribution is often assumed in a factorized form with the Gaussian distribution in $k_{\perp}$ as

$$
f^{u+d}\left(x, \boldsymbol{k}_{\perp}\right)=f^{u+d}(x) \frac{1}{\pi\left\langle k_{\perp}^{2}\right\rangle} e^{-k_{\perp}^{2} /\left\langle k_{\perp}^{2}\right\rangle},
$$


with $\left\langle k_{\perp}^{2}\right\rangle \simeq 0.25 \mathrm{GeV}^{2}$. Then, it may be of some interest to compare the predictions of the CQSM for the TMD quark distribution $f^{u+d}\left(x, \boldsymbol{k}_{\perp}\right)$ with this factorized ansatz. We show in Fig, 4 the contour plot for the isoscalar unpolarized TMD quark distribution function $f^{u+d}\left(x, \boldsymbol{k}_{\perp}\right)$. The left panel corresponds to the prediction of the CQSM, while the right panel to the factorized form given by (26) . (For the integrated distribution $f^{u+d}(x)$ in the factorized form, we use the prediction of the CQSM, i.e. it is obtained from the CQSM prediction for $f^{u+d}\left(x, \boldsymbol{k}_{\perp}\right)$ after integration over $k_{\perp}$.) One clearly sees significant difference between the behaviors of the two distributions especially in the lower $x$ region, $x<0.4$, where the magnitude of the TMD distribution is dominantly large.
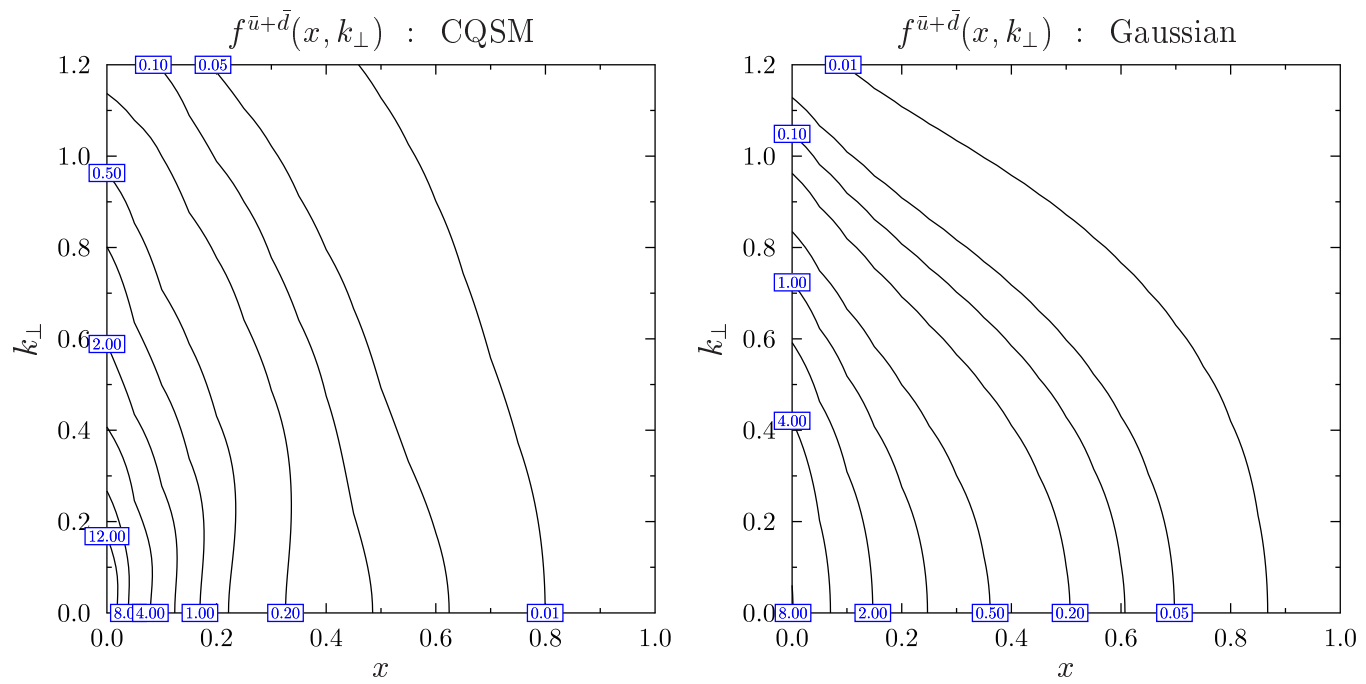

FIG. 5: The contour plot for the isoscalar unpolarized TMD antiquark distribution $f^{\bar{u}+\bar{d}}\left(x, k_{\perp}\right)$. The meaning of the curves is the same as in Fig, 4

Fig.5 show a similar comparison for antiquark distribution $f^{\bar{u}+\bar{d}}\left(x, \boldsymbol{k}_{\perp}\right)$. One again observes significant difference between the prediction of the CQSM and the simple parametrization (26). According to the prediction of the CQSM, the TMD antiquark distribution with lower $x$ is seen to extend over higher $k_{\perp}$ region, as compared with the schematic form. At any rate, the analysis above indicates that the frequently used assumption of factorization in $x$ and $k_{\perp}$ is most probably violated. Later, this statement can be made a little more quantitative through the investigation of the average transverse momentum square of quarks and 
antiquarks in dependence of the longitudinal momentum fraction $x$. But, before doing it, here demonstrate realistic nature of the predictions of the CQSM, through the analysis of the empirically well-known integrated quark and antiquark distributions, the information of which is hidden in the TMD distribution $f^{u+d}\left(x, \boldsymbol{k}_{\perp}\right)$.
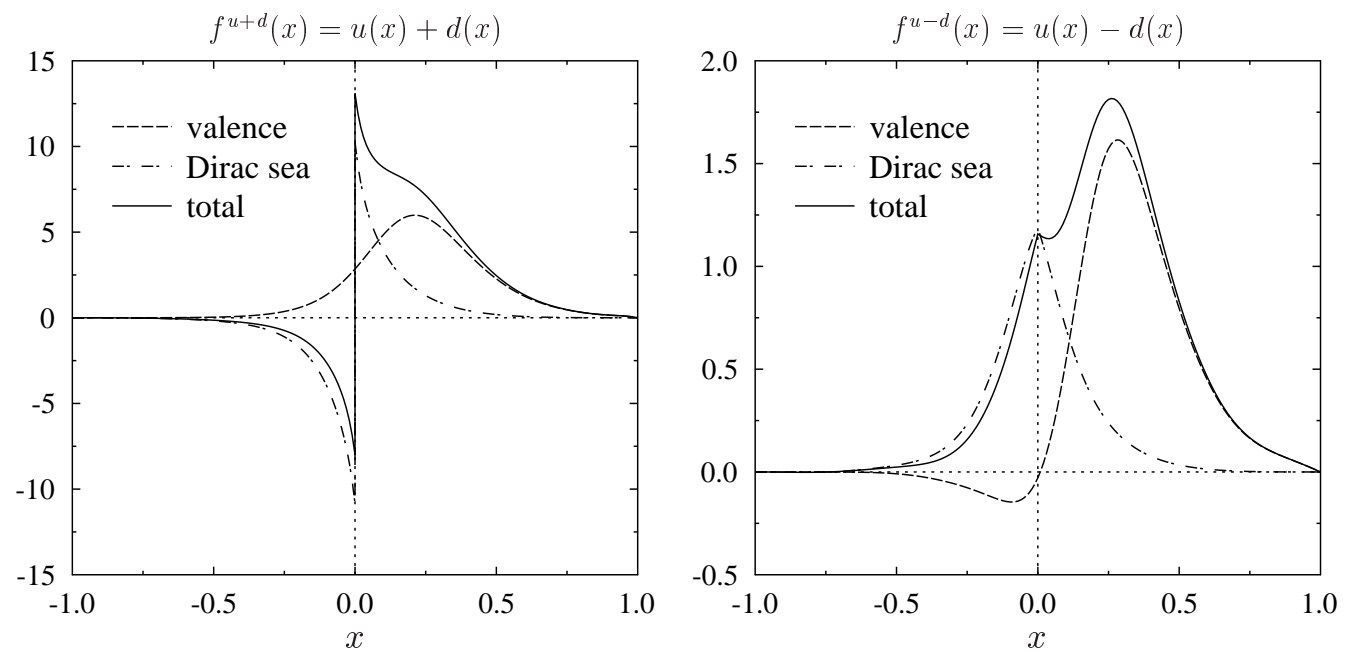

FIG. 6: The integrated unpolarized quark and antiquark distribution function $f^{u+d}(x)$ with isoscalar combination obtained from the corresponding TMD distribution via (27) (left panel). The meaning of the curves is the same as in Fig.1. Shown in the right panel is the unpolarized quark and antiquark distribution with isovector combination, which has been evaluated directly, i.e. without reference to the TMD distribution, in the previous papers.

To demonstrate the reliability of the CQSM predictions for the unpolarized TMD quark distributions shown in Fig.1 and Fig.2, we evaluate from $f^{u+d}\left(x, \boldsymbol{k}_{\perp}\right)$ the corresponding integrated quark distributions through the relation,

$$
f^{u+d}(x)=\int d^{2} \boldsymbol{k}_{\perp} f^{u+d}\left(x, \boldsymbol{k}_{\perp}\right)
$$

The answer is shown in the left panel of Fig 6 , which nicely reproduces the previous results for $u(x)+d(x)$ calculated directly without referring to the TMD quark distribution [48]. (A finer look however reveals that our new result for $f^{u+d}(x)$ shows a slight enhancement in the magnitude of the Dirac-sea contribution as compared with our old result shown in Fig.1(a) of [48], for which the check of the model space dependence, i.e. the $D \rightarrow \infty$ and $k_{\max } \rightarrow \infty$ limit, was not satisfactory enough. The new result for the $\bar{d}(x) / \bar{u}(x)$ ratio to be shown 
below is a little sensitive to this change of the isoscalar unpolarized parton distribution.) One confirms again that the proper introduction of the Dirac-sea contribution is essential for reproducing the positivity condition of the unpolarized antiquark distribution. One also sees that, in the lower $x$ region, even the quark distribution is dominated by the contributions of Dirac-sea quarks. If one compares this figure of $f^{u+d}(x)$ with the Fig 2 for TMD distribution $f^{u+d}\left(x, \boldsymbol{k}_{\perp}\right)$ especially at $x=1.0 \times 10^{-6}$ and $x=0.1$, one can confirm that the longer range tail in $k_{\perp}$ of the Dirac-sea contribution is an important factor leading to its strong enhancement in the small $x$ region, which we observe for the integrated distribution $f^{u+d}(x)$. In the right panel of Fig, $\llbracket$, we also show the previously calculated isovector unpolarized quark distribution $f^{u-d}(x)$, since it is necessary for the following comparison of the $\bar{d}(x) / \bar{u}(x)$ ratio.
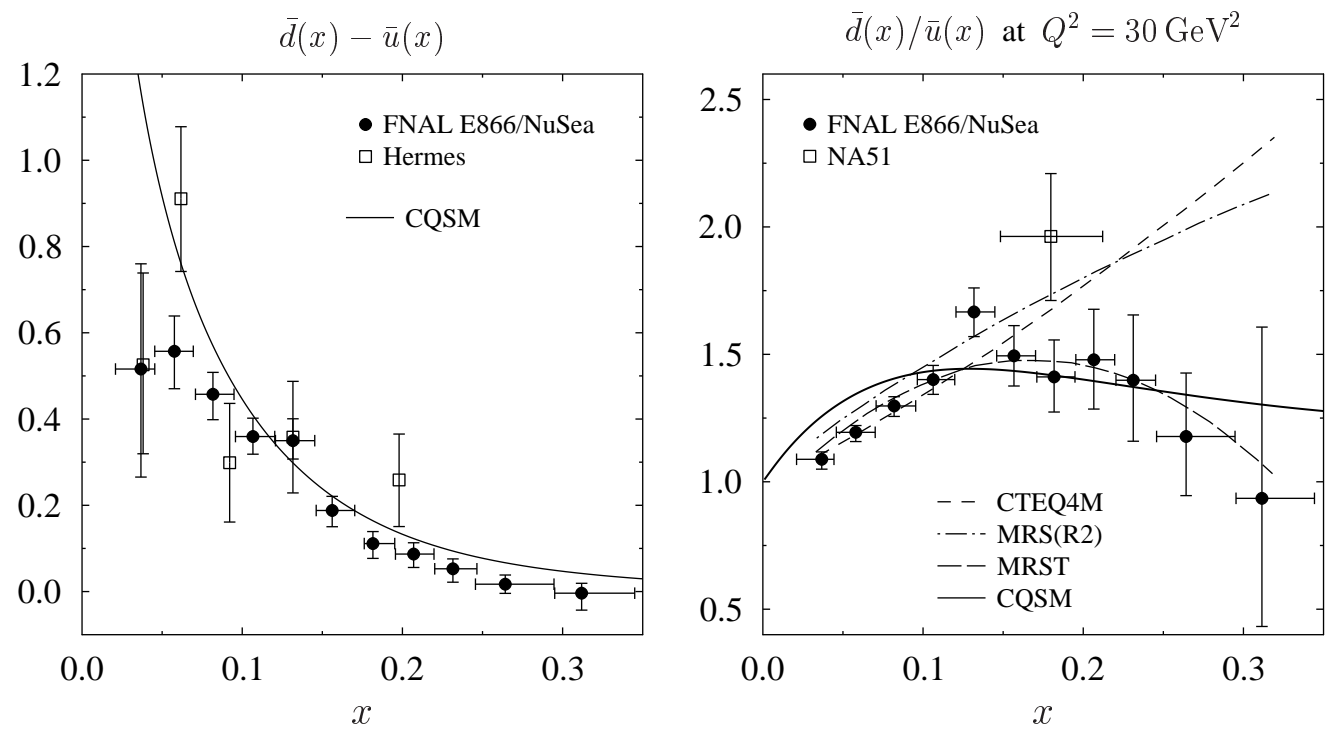

FIG. 7: The CQSM predictions for the difference function $\bar{d}(x)-\bar{u}(x)$ in the proton in comparison with the FNAL [61] and HERMES data [77] (left panel), and for the ratio $\bar{d}(x) / \bar{u}(x)$ in comparison with the FNAL (Fermi National Accelerator Laboratory) 61] and NA51 data [60] (right panel). The curves labeled CTEQ4M, MRS(R2) and MRST are phenomenological PDF fits to the data before and after the E866 measurement.

In order to demonstrate realistic nature of the CQSM predictions for the antiquark distributions, we show in Fig[7its predictions for the difference $\bar{u}(x)-\bar{d}(x)$ and the ratio $\bar{d}(x) / \bar{u}(x)$ in comparison with the corresponding empirical data. As in the previous works [47], [48], the 
theoretical predictions for the distribution functions at the high energy scales are obtained by solving the DGLAP equations at the next-to-leading oder, with the predictions of the CQSM as initial scale distributions given at the scale $Q_{i n i}^{2}=0.30 \mathrm{GeV}^{2}$. (Here, the suffix "ini" should be understood as an abbreviation of the word "initial".) A good agreement observed in the left panel means that the model reproduces the famous NMC observation, i.e. the dominance of the $\bar{d}$-sea over the $\bar{u}$-sea inside the proton, without any artificial finetuning. Shown in in the right panel of Fig.7 is the CQSM prediction for the ratio $\bar{d}(x) / \bar{u}(x)$ at $Q^{2}=30 \mathrm{GeV}^{2}$ in comparison with the old NA51 data [60] and the newer E866 data extracted from the neutrino scatterings. The new MRST parton distribution function (PDF) fit including the E866 data 61] together with the older CTEQ4M and MRS(R2) PDF fits are also shown for reference. One sees that the prediction of the CQSM is qualitatively consistent with the E866 data as well as the new MRST fit. We emphasize again that it is a completely parameter free prediction of the model.

Now, after demonstrating that the CQSM well describes the fundamental physics of unpolarized sea quark distributions, we come back to its predictions on the unpolarized TMD distributions for quarks and antiquarks. From the already-given unpolarized TMD distributions $f^{u+d}\left(x, \boldsymbol{k}_{\perp}\right)$ as functions of $x$ and $k_{\perp}^{2}$, it is straightforward to evaluate the average transverse momentum of quarks and antiquarks as a function of $x$ :

$$
\left\langle k_{\perp}^{2}(x)\right\rangle=\frac{\int d^{2} \boldsymbol{k}_{\perp} k_{\perp}^{2} f^{u+d}(x, \boldsymbol{k})}{\int d^{2} \boldsymbol{k}_{\perp} f^{u+d}(x, \boldsymbol{k})} .
$$

The resultant $\left\langle k_{\perp}^{2}(x)\right\rangle$ is shown by filled circles in Fig, The solid curve here is a smooth fit to the numerical results by an 8 th-order polynomial as

$$
\left\langle k_{\perp}^{2}(x)\right\rangle=\sum_{n=0}^{8} c_{n} x^{n}
$$

where

$$
\begin{aligned}
& c_{0}=0.311122, \quad c_{1}=-0.536064, \quad c_{2}=-2.4806, \\
& c_{3}=18.7331, \quad c_{4}=-41.7892, \quad c_{5}=40.5805, \\
& c_{6}=-11.3483, \quad c_{7}=-7.48546, \quad c_{8}=4.26044,
\end{aligned}
$$


for $x>0$, while

$$
\begin{aligned}
& c_{0}=0.311122, \quad c_{1}=-0.805606, \quad c_{2}=14.9032, \\
& c_{3}=114.34, \quad c_{4}=376.97, \quad c_{5}=693.994, \\
& c_{6}=733.782, \quad c_{7}=415.798, \quad c_{8}=97.7558,
\end{aligned}
$$

for $x<0$.

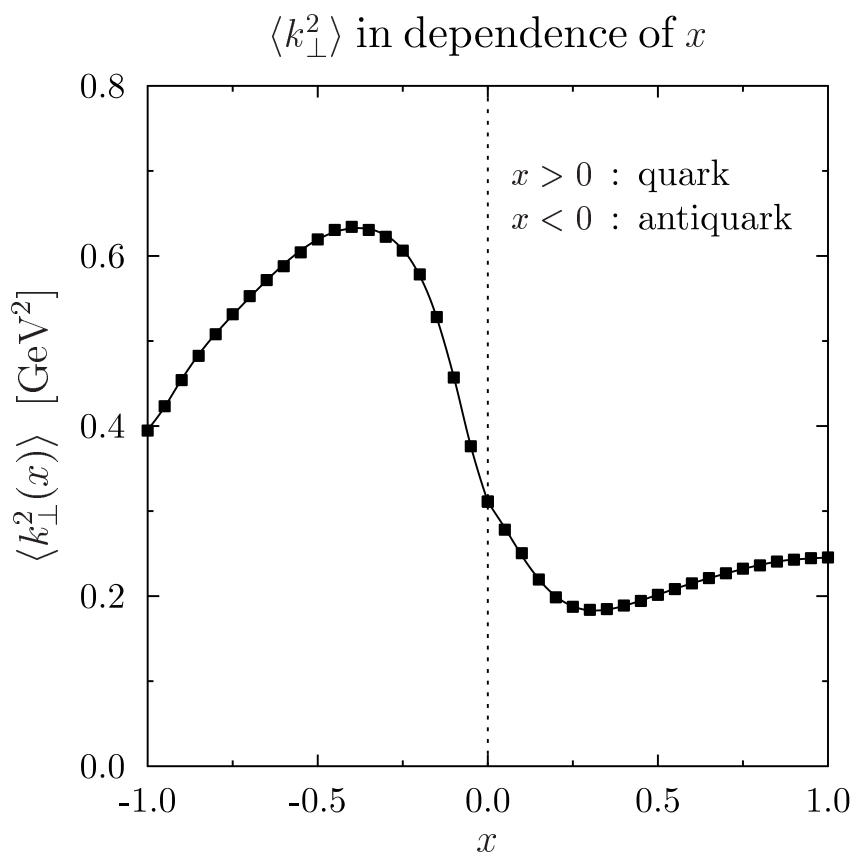

FIG. 8: The CQSM prediction for the average transverse momentum square of quarks $(x>0)$ and antiquarks $(x<0)$ as a function of the longitudinal momentum fraction $x$.

One clearly sees that the average transverse momentum square is strongly dependent on the longitudinal momentum fraction $x$ of quarks and antiquarks. This reconforms that the frequently used assumption of factorization in the variables $x$ and $k_{\perp}$ is significantly broken. (The plausible breakdown of the usual Gaussian ansatz for a factorized $k_{\perp}$ dependence was also indicated in the model calculation by Pasquinni et al. [27]) Very recently, the first lattice QCD study of the transverse momentum distributions of quarks in the nucleon has been reported [36]. The validity of factorization in $x$ and $k_{\perp}$ was examined, and it has been concluded that the factorization hypothesis holds within the statistics of the simulation in sharp contrast to our present analysis based on the CQSM. In our opinion, the results of the 
lattice QCD simulation at the present level cannot be taken too seriously by several reasons. First, the simulation is preliminary in that it was performed in the fictitious heavy-pion region around $m_{\pi} \simeq 500 \mathrm{MeV}$. What is lacking here is the nonperturbative chiral dynamics of light quarks, which is the core of the celebrated NMC observation, or the physics of Gottfried sum. Second, the test of factorization is far from persuasive, since the evaluation of full $x$-dependence is beyond the scope of the presently-known algorithms of lattice QCD.

Turning back to the $x$-dependence of the average transverse momentum square of quarks and antiquarks, we continue to analyze the prediction of the CQSM. Let us first look into the positive $x$ region corresponding to the quark distribution. Very strangely, the average transverse momentum square has a minimum around $x \sim 0.25$, and it increases as $x$ becomes larger. This feature can be understood as follows. Around $x \sim(0.2-0.3)$, the integrated quark distribution $f^{u+d}(x)$ has a peak, which is dominated by the contribution of the valencelevel quarks. This means that the relative importance of Dirac-sea quarks, which has higher $k_{\perp}$ components, as compared to the valence-level quark, is smallest around there, which explains the above-mentioned feature of $\left\langle k_{\perp}^{2}(x)\right\rangle$ as a function of $x$. One may also notice that the average transverse momentum square around $x=0$ is fairly large, which indicates that the quarks (and antiquarks) with small longitudinal momentum fraction would carry sizable amount of orbital angular momentum along the $z$-axis, i.e. along the the direction of nucleon spin. We recall that this observation is consistent with the results of [62], in which a direct calculation of quark orbital-angular-momentum distributions was carried out. (See also the analyses of the quark orbital angular momentum from the viewpoint of the generalized parton distribution functions [63] - [66].)

Another unique feature of the CQSM prediction is that the magnitude $\left\langle k_{\perp}^{2}(x)\right\rangle$ is much larger in the negative $x$ region, corresponding to the antiquarks. Another way of convincing this feature is to compare the two quantities defined below :

$$
\begin{aligned}
\left\langle k_{\perp}^{2}\right\rangle^{Q} & \equiv \frac{\int_{0}^{1} d x\left\langle k_{\perp}^{2}(x)\right\rangle f^{u+d}(x)}{\int_{0}^{1} d x f^{u+d}(x)} \\
\left\langle k_{\perp}^{2}\right\rangle^{\bar{Q}} & \equiv \frac{\int_{-1}^{0} d x\left\langle k_{\perp}^{2}(x)\right\rangle f^{u+d}(x)}{\int_{-1}^{0} d x f^{u+d}(x)}=\frac{\int_{0}^{1} d x\left\langle k_{\perp}^{2}(x)\right\rangle f^{\bar{u}+\bar{d}}(x)}{\int_{0}^{1} d x f^{\bar{u}+\bar{d}}(x)},
\end{aligned}
$$

which represent the average transverse momentum square for quarks and antiquarks, respec- 
tively. Numerically, we find that

$$
\begin{aligned}
& \left\langle k_{\perp}^{2}\right\rangle^{Q}=0.224 \mathrm{GeV}^{2}, \\
& \left\langle k_{\perp}^{2}\right\rangle^{\bar{Q}}=0.445 \mathrm{GeV}^{2},
\end{aligned}
$$

which clearly shows that the average transverse momentum of antiquarks is much larger than that of quarks. We can also estimate the average transverse momentum square of quarks and antiquarks altogether from

$$
\left\langle k_{\perp}^{2}\right\rangle^{Q+\bar{Q}} \equiv \frac{\int_{-1}^{1} d x\left\langle k_{\perp}^{2}(x)\right\rangle f^{u+d}(x)}{\int_{-1}^{1} d x f^{u+d}(x)},
$$

which gives

$$
\left\langle k_{\perp}^{2}\right\rangle^{Q+\bar{Q}}=0.266 \mathrm{GeV}^{2} .
$$

This value of average transverse momentum square is remarkably close to that used in the phenomenological analysis of the semi-inclusive reactions [23]. Note, however, that the average transverse momentum is a scale dependent quantity, which is believed to grow as $Q^{2}$ increases. The predictions of the CQSM corresponds to the scale $Q^{2} \sim(0.30-0.40) \mathrm{GeV}^{2}$ [47],[48], while the phenomenological analysis in [23] should correspond to somewhat higher energy scale. In any phenomenological analysis, one must keep in mind this scale dependent nature of the average transverse momentum square of quarks and antiquarks.

Before ending this section, we make a short comment on the so-called transversecoordinate representation of the TMD parton distributions. The $Q^{2}$-evolution of the TMD parton distributions or the unintegrated parton distributions is described by the CataniCiafaloni-Fiorani-Marchesini (CCFM) equations [67]-[69]. A nice feature of the CCFM equation at the leading order is that they reproduce the convectional leading-order DokshitzerGribov-Lipatov-Altarelli-Parisi (DGLAP) equations for the integrated distributions. The CCFM equations are known to take a particularly simple structure if one introduces the so-called transverse-coordinate representation of the parton distributions [70]-[73]. To explain it, we represent below the TMD quark (or antiquark) distribution of flavor $a$ as $f^{a}\left(x, k_{\perp}, Q^{2}\right)$ with $k_{\perp}=\left|\boldsymbol{k}_{\perp}\right|$, where the dependence on the scale $Q^{2}$ is also shown explicitly. The transverse-coordinate representation $\bar{f}^{a}\left(x, b, Q^{2}\right)$ of a quark (or an antiquark) of flavor $q$ is introduced as the two-dimensional Fourier transform of the corresponding TMD distribution $f^{a}\left(x, k_{\perp}, Q^{2}\right)$ :

$$
\bar{f}^{a}\left(x, b, Q^{2}\right) \equiv \int d^{2} \boldsymbol{k}_{\perp} e^{i \boldsymbol{b} \cdot \boldsymbol{k}_{\perp}} f^{a}\left(x, k_{\perp}, Q^{2}\right),
$$


where $\boldsymbol{b}$ is the transverse coordinate vector. Clearly, at $b=0$, the function $\bar{f}^{a}\left(x, b, Q^{2}\right)$ reduces to the familiar integrated distribution $f^{a}\left(x, Q^{2}\right)$. The greatest advantage of using the transverse-coordinate representation is that the corresponding evolution equations for the unintegrated distributions, $\bar{f}_{N S}\left(x, b, Q^{2}\right), \bar{f}_{S}\left(x, b, Q^{2}\right)$ and $\bar{f}_{g}\left(x, b, Q^{2}\right)$, become diagonal in the transverse coordinate $b$. Here, just for illustration, we show the evolution equation for the nonsinglet (NS) distribution. It takes the form [74], [75] :

$$
\begin{aligned}
Q^{2} \frac{\partial^{2}}{\partial Q^{2}} \bar{f}_{N S}\left(x, b, Q^{2}\right) & =\frac{\alpha_{S}\left(Q^{2}\right)}{2 \pi} \int_{0}^{1} d z P_{q q}(z) \\
\times & \left.\times \Theta(z-x) J_{0}[(1-z) Q b] \bar{f}_{N S}\left(\frac{x}{z}, b, Q^{2}\right)-\bar{f}_{N S}\left(x, b, Q^{2}\right)\right\}
\end{aligned}
$$

which certainly is diagonal in the variable $b$. Once these evolution equations are solved in a similar way as the standard DGLAP equations, the average transverse momentum square for a given distribution at any $Q^{2}$ can be evaluated from

$$
\left\langle k_{\perp}^{2}\left(x, Q^{2}\right)\right\rangle^{a}=\frac{\int d^{2} \boldsymbol{k}_{\perp} k_{\perp}^{2} f^{a}\left(x, k_{\perp}, Q^{2}\right)}{\int d^{2} \boldsymbol{k}_{\perp} f^{a}\left(x, k_{\perp}, Q^{2}\right)}=-\left.4 \frac{\frac{d}{d b^{2}} \bar{f}^{a}\left(x, b, Q^{2}\right)}{\bar{f}^{a}\left(x, b, Q^{2}\right)}\right|_{b=0} .
$$

The transverse-coordinate representation of the unintegrated parton distribution discussed above should not be confused with more popular impact-parameter dependent parton distributions introduced by Burkardt [5], although there should be strong correlation between them. The simplest impact-parameter dependent parton distribution function $f^{a}\left(x, b_{\perp}\right)$ is the two dimensional Fourier transform of the unpolarized generalized parton distribution function $H^{a}(x, \xi, t)$ with zero skewdness parameter $\xi=0$ :

$$
f^{a}\left(x, b_{\perp}\right)=\int \frac{d^{2} \boldsymbol{\Delta}}{(2 \pi)^{2}} e^{-i \boldsymbol{\Delta} \cdot \boldsymbol{b}_{\perp}} H^{a}\left(x, \xi=0, t=-\boldsymbol{\Delta}^{2}\right) .
$$

We recall that the frequently used parametrization [76] of $H^{a}(x, \xi=0, t)$ is of nonfactorizable form in $x$ and $t$ as

$$
H^{a}(x, \xi=0, t)=f^{a}(x) \exp \left[\beta^{a}(x) t\right],
$$

with

$$
\beta^{a}(x)=\left[\alpha^{\prime} \ln (1 / x)+B_{a}\right](1-x)^{3}+A_{a} x(1-x)^{2},
$$

which means that $f^{a}\left(x, b_{\perp}\right)$ is also non-factorizable in $x$ and $b_{\perp}$. This in turn strongly indicates that the transverse-coordinate representation of the unintegrated parton distributions, and consequently, the TMD parton distributions are most likely to be non-factorizable in the variables $x$ and $k_{\perp}$, as our predictions based on the CQSM shows explicitly. 


\section{CONCLUSION}

To conclude, we have reported the first calculation of the simplest but most fundamental TMD parton distribution in the nucleon, i.e. the unpolarized TMD quark and antiquark distributions with isoscalar combination, within the framework of the CQSM. The realistic nature of the CQSM predictions was demonstrated by showing that the integrated unpolarized distribution $f^{u+d}(x)$, obtained from the corresponding TMD distribution $f^{u+d}\left(x, \boldsymbol{k}_{\perp}\right)$, is consistent with the available empirical information on the light-flavor unpolarized parton distributions. It was found that the predicted average transverse momentum square of quarks and antiquarks depends strongly on their longitudinal momentum fraction $x$. We also estimate the average transverse momentum of quarks and antiquarks separately to find, somewhat unexpectedly, that $\left\langle k_{\perp}^{2}\right\rangle^{Q} \simeq 0.224 \mathrm{GeV}^{2}$ and $\left\langle k_{\perp}^{2}\right\rangle^{\bar{Q}} \simeq 0.445 \mathrm{GeV}^{2}$, that is, the antiquarks have much higher average transverse momentum than the quarks. On the other hand, the average momentum transfer of quarks and antiquarks altogether turns out to be $\left\langle k_{\perp}^{2}\right\rangle^{Q+\bar{Q}} \simeq 0.266 \mathrm{GeV}^{2}$, which is order of magnitude consistent with the recent phenomenological analysis, although we must be careful about the fact that the average transverse momentum square is a $Q^{2}$-dependent quantity.

[1] J.C. Collins and D.E. Soper, Nucl. Phys. B194, 445 (1982).

[2] SMC Collaboration, A. Bravar et al., Nucl. Phys. A666, 314 (2000).

[3] HERMES Collaboration, A. Airapetian et al., Phys. Rev. Lett. 94, 012002 (2005).

[4] COMPASS Collaboration, V.Y. Alexakhin et al., Phys. Rev. Lett. 94, 202002 (2005).

[5] M. Burkardt, Phys. Rev. D 62, 071503(R) (2000).

[6] J.P. Ralston and B. Pire, Phys. Rev. D 66, 111501 (2002).

[7] M. Diehl, Eur. Phys. J. C25, 223 (2002).

[8] A.V. Belitsky, X. Ji, and F. Yuan, Phys. Rev. D 69, 074014 (2004).

[9] P.J. Mulders and R.D. Tangelman, Nucl. Phys. B461, 197 (1996) ; B484, 538(E) (1997).

[10] K. Goeke, A. Metz, and M. Schlegel, Phys. Lett. B618, 90 (2005).

[11] A. Bacchetta, M. Diehl, K. Goeke, A. Mettz, P.J. Mulders, and M. Schlegel, J. High Energy Phys. 0702, 093 (2007). 
[12] D.W. Sivers, Phys. Rev. D 41, 83 (1990).

[13] D.W. Sivers, Phys. Rev. D 43, 261 (1991).

[14] D. Boer and P.J. Mulders, Phys. Rev. D 57, 5780 (1998).

[15] S.J. Brodsky, D.S. Huang, and I. Schmidt, Phys. Lett. B530, 99 (2002).

[16] J.C. Collins, Phys. Lett. B536, 43 (2002).

[17] J.C. Collins, Phys. Rev. D 57, 3051 (1998).

[18] X. Ji and F. Yuan, Phys. Lett. B543, 66 (2002).

[19] A.V. Belitsky, X. Ji, and F. Yuan, Nucl. Phys. B656, 165 (2003).

[20] J.C. Collins, Acta. Phys. Polon. B34, 3103 (2003).

[21] C.J. Bomhof, P.J. Mulders, and F. Pijlman, Eur. Phys. J. C47, 147 (2006).

[22] C.J. Bomhof and P.J. Mulders, Nucl. Phys. B795, 409 (2008).

[23] M. Anselmino, M. Boglione, J.C. Collins, U. D'Alesio, A.V. Efremov, K. Goeke, A. Kotzinian, S. Menzel, A. Metz, F. Murgia, A. Prokudin, P. Schweitzer, W. Vogelsang, and F. Yuan, arXiv:hep-ph/0511017 (2005).

[24] B. Zhang, L. Zhun, B.-Q. Ma, and I. Schmidt, Phys. Rev. D 77, 054011 (2008).

[25] A. Bacchetta, F. Conti, and M. Radici, Phys. Rev. D 78, 074010 (2008).

[26] R. Jakob, P.J. Mulders, and J. Rodrigues, Nucl. Phys. A626, 937 (1997).

[27] B. Pasquini, S. Cazzaniga, and S. Boffi, Phys. Rev. D 78, 034025 (2008).

[28] L.P. Gamberg, G.R. Goldstein, and K.A. Oganessyan, Phys. Rev. D 67, 071504(R) (2003).

[29] D. Boer, S.J. Brodsky, and D.S. Hwang, Phys. Rev. D 67, 054003 (2003).

[30] L.P. Gamberg, G.R. Goldstein, and M. Schlegel, Phys. Rev. D 77, 094016 (2008).

[31] A. Bacchetta, A. Schäfer, and J.-J. Yang, Phys. Lett. B578, 109 (2004).

[32] F. Yuan, Phys. Lett. B575, 45 (2003).

[33] I.O. Cherednikov, U. D’Alesio, N.I. Kochelev, and F. Murgia, Phys. Lett. B642, 39 (2006).

[34] A. Courtoy, S. Scopetta, V. Vento, arXiv:0811.1191[hep-ph].

[35] A. Courtoy, F. Fratini, S. Scopetta, and V. Vento, Phys. Rev. D 78, 034002 (2008).

[36] B.U. Musch, P. Hägler, A. Schäfer, D.B. Renner, and J.W. Negele, arXiv:0811.1536 [hep-lat] (2008).

[37] R.M. Davidson and E. Ruiz Arriola. Phys. Lett. B348, 163 (1995).

[38] H. Weigel, E. Ruiz Arriola, and L. Gamberg, Nucl. Phys. B560, 383 (1999).

[39] E. Ruiz Arriola, Acta Phys. Polon. B33, 4443 (2002). 
[40] D.I. Diakonov, V.Yu. Petrov, and P.V. Pobylitsa, Nucl. Phys. B306, 809 (1988).

[41] M. Wakamatsu and H. Yoshiki, Nucl. Phys. A524, 561 (1991).

[42] D.I. Diakonov, V.Yu. Petrov, P.V. Pobylitsa, M.V. Polyakov, and C. Weiss, Nucl. Phys. B480, 341 (1996).

[43] D.I. Diakonov, V.Yu. Petrov, P.V. Pobylitsa, M.V. Polyakov, and C. Weiss, Phys. Rev. D56, 4069 (1997).

[44] H. Weigel, L. Gamberg, and H. Reinhardt, Phys. Lett. B399, 287 (1997).

[45] H. Weigel, L. Gamberg, H. Reinhardt, Phys. Rev. D 55, 6910 (1997).

[46] M. Wakamatsu and T. Kubota, Phys. Rev. D 57. 5755 (1998).

[47] M. Wakamatsu and T. Kubota, Phys. Rev. D 60. 034020 (1999).

[48] M. Wakamatsu, Phys. Rev. D 67, 034005 (2003) ; Phys. Rev. D 67, 034006 (2003).

[49] M. Wakamatsu, Phys. Rev. D 46, 3762 (1992).

[50] P.V. Pobylitsa, M.V. Polyakov, K. Goeke, T. Watabe, and C. Weiss, Phys. Rev. D 59, 034024 (1999).

[51] S. J. Brodsky, arXiv:0901.0781[hep-ph] (2009).

[52] P.V. Pobylitsa, arXiv:hep-ph/0301236 (2003).

[53] S. Kahana and G. Ripka, Nucl. Phys. A429, 462 (1984).

[54] S. Kahana, G. Ripka, and V. Soni, Nucl. Phys. A415, 351 (1984).

[55] T. Kubota, M. Wakamatsu, and T. Watabe, Phys. Rev. D 60, 014016 (1999).

[56] A.V. Efremov and P. Schweitzer, JHEP 0308:006 (2003).

[57] M. Wakamatsu and Y. Ohnishi, Phys. Rev. D 67, 114011 (2003)

[58] Y. Ohnishi and M. Wakamatsu, Phys. Rev. D 69, 114002 (2004).

[59] C. Cebulla, J. Ossmann, P. Schweitzer, and D. Urbano, Acta Phys. Polon. B39, 609 (2008).

[60] A. Baldit et al., Phys. Lett. B332, 244 (1994).

[61] E866 Collaboration, E.A. Hawker et al., Phys. Rev. Lett. 80, 3715 (1998).

[62] M. Wakamatsu and T. Watabe, Phys. Rev. D 62, 054009 (2000).

[63] J. Ossmann, M.V. Polyakov, P. Schweitzer, D. Urbano, and K. Goeke, Phys. Rev. D 71, 034011 (2005).

[64] M. Wakamatsu and H. Tsujimoto, Phys. Rev. D 71, 074001 (2005).

[65] M. Wakamatsu and Y. Nakakoji, Phys. Rev. D 74, 054006 (2006).

[66] M. Wakamatsu and Y. Nakakoji, Phys. Rev. D 77, 074011 (2008). 
[67] M. Ciafaloni, Nucl. Phys. B296, 49 (1988).

[68] S. Catani, F. Fiaorani, and Marchesini, Phys. Lett. B234, 339 (1990) ; Nucl. Phys. B336, 18 (1990).

[69] A. Bassetto, M. Ciafaloni, and G. Marchesini, Nucl. Phys. B163, 477 (1980).

[70] J. Kodaira and L. Trentadue, Phys. Lett. B112, 66 (1982).

[71] Y.I. Dokshitzer, D.I. Diakonov, and S.I. Troyan, Phys. Lett. B79, 269 (1978).

[72] J. Kwieciński, Acta. Phys. pol. B33, 1809 (2002).

[73] A. Gawron and J. Kwieciński, Acta. Phys. B34, 133 (2003).

[74] A. Gawron, J. Kwieciński, and W. Broniowski, Phys. Rev. D 68, 054001 (2003).

[75] E. Ruiz Arriola and W. Broniowski, Phys. Rev. D 70, 034012 (2004).

[76] M. Diehl, Th. Feldmann, R. Jakob, and P. Kroll, Eur. Phys. J. C39, 1 (2005).

[77] HERMES Collaboration, K. Ackerstaff et al., Phys. Rev. Lett. 81, 5519 (1998). 